\title{
Microfluidics of temperature-responsive partially miscible binary liquid mixtures
}

\author{
Maximiliano J. Fornerod ${ }^{\mathrm{a}}$, Esther Amstad ${ }^{\mathrm{b}}$, Stefan Guldin ${ }^{\mathrm{a}}$.
}

Two-phase liquid-liquid microfluidics relies on the intricate control over the fluid properties of liquid mixtures. Herein, we report on the use of partially miscible binary liquid mixtures that lend their microfluidic properties from a highly temperature-sensitive mixing and phase separation behaviour. For a blend composed of the thermotropic liquid crystal 4Cyano-4'-pentylbiphenyl (5CB) and methanol, mixing at temperatures above the upper critical solution temperature (UCST) leads to a uniform single phase while partial mixing can be achieved at temperatures below the UCST. Thermally-driven phase separation inside the microfluidic channels results in the formation of very regular phase arrangements, namely in droplets, plug, slug and annular flow. We map different flow regimes and relate findings to the role of viscous, interfacial and inertial forces. As the interfacial tension of the mixture and the dynamic viscosity of the separated phases are inversely proportional to temperature, different flow regimes can be achieved at constant channel architecture and flow rate. $A$ consistent behaviour is observed for a binary liquid mixture with lower critical solution temperature, namely 2,6-lutidine and water. This temperature-responsive approach to microfluidics is an interesting candidate for multi-stage processes, selective extraction and sensing applications.

\section{Introduction}

Microfluidics offers several advantages for fluid handling such as small sample volumes, the possibility to separate and detect substances with high sensitivity, as well as compatibility with low-cost and portable device architectures [1]. Consequently, microfluidic processes are used in a broad range of fields, including drug development [2], point-of-care diagnostics [3] bioanalysis [4], systems biology [5] , cell biology [6], chemical synthesis [7] and production of foams and emulsions [8]. These applications usually involve the use of either miscible or immiscible fluids. For processes that require liquid-liquid mass transfer, immiscible mixtures are typically chosen, and phase transfer then relies on the diffusion of target molecules across the liquid-liquid interface [9] [10] [11] [12]. Different approaches have been developed to enhance transfer rates, either based on passive mixers, such as stream splitters [13] or ridges [14] [15], or active mixers, including bubblers [16], magnetic bar micromixers [9] [17] and magnetic fields to induce diffusion [18].

In contrast, the use of temperature manipulation to achieve control over microfluidic properties has only been explored to a somewhat limited extend. Successful examples include the variation of temperature to enhance convection, and thus, mixing of miscible components [19] as well as the temperature-induced tuning of interfacial tension and viscosity of immiscible mixtures [20] [21] [22] [23].

The class of binary liquid compositions that exhibit temperature-dependent miscibility are referred to as regular solutions. For liquid-liquid mixtures with an upper critical solution temperature (UCST), the solution is single phase above the temperature threshold. Cooling below the coexistence curve in the corresponding phase diagram, typically described by temperature $T$ and composition $\phi$, leads to phase separation. The temperature response is opposite for a binary liquid mixture with a lower critical solution temperature (LCST). A comprehensive overview of conventional binary liquid mixtures and their critical solution temperatures can be found in a monograph by Francis [24]. Despite the broad library of suitable liquid-liquid combinations, to-date regular solutions have received little attention in a microfluidic environment.

In this work, we introduce the use of temperature-responsive partially miscible binary liquid mixtures for microfluidic applications. Our material system of interest is a 50/50 v/v blend of the thermotropic liquid crystal 4-Cyano-4'pentylbiphenyl (5CB) and methanol (MeOH) with an UCST of $24^{\circ} \mathrm{C}$. Using microfluidic devices, we investigate the influence of the temperature on the miscibility, interfacial tension, and viscosity of the mixture. We carefully mix and phase separate the liquid blend and relate the observed formation of twophase fluid systems to the role of the interfacial, viscous, and inertial forces. Finally, we compare findings to a binary liquid mixture with a lower critical solution temperature, namely water and 2,6-lutidine to probe the general applicability of our approach. 


\section{Experimental}

\section{Reagents}

The liquid crystal 4-Cyano-4'-pentylbiphenyl (5CB) (CAS 4081708-1, 99.5\%) was purchased from Synthon Chemicals. $\mathrm{MeOH}$ (CAS 67-56-1, HPLC grade) and 2,6-lutidine (CAS 108-48-5, 98\%) were obtained from Sigma Aldrich. All chemicals were used as received.

\section{Mixing characterisation}

Mixing of $\mathrm{MeOH}$ and $5 \mathrm{CB}$ was studied in a microfluidic chip with a channel width of $300 \mu \mathrm{m}$ and a channel depth of $120 \mu \mathrm{m}$ containing static micromixer with staggered oriented ridges (Chemtrix BV, chip no. 3227). Pure $\mathrm{MeOH}$ and pure 5CB were infused onto the chip with a syringe pump (Cronus, Sigma 1110) at a flow rate of $5 \mu \mathrm{l} / \mathrm{min}$ for each stream. The liquid reservoirs were kept at room temperature. The temperature of the microfluidic device was controlled with a custom-built Peltierbased chip holder (see Supplementary Information, Figure S1). A k-type thermocouple was attached with an adhesive tape (DuPont, Kapton) to the surface of the microfluidic chip in order to verify the temperature of the setup. The mixing was studied for a range of temperatures from $40^{\circ} \mathrm{C}$ to $12^{\circ} \mathrm{C}$. Images of the micromixer were taken with a digital camera (FLIR, Chamaleon3) mounted on a stereo microscope (VWR).

\section{Interfacial tension}

The interfacial tension was measured via the pendant drop method. $2 \mathrm{ml}$ of a 50/50 v/v mixture of $\mathrm{MeOH}$ and $5 \mathrm{CB}$ was prepared and mixed at $40^{\circ} \mathrm{C}$ in a temperature-controlled quartz cuvette (Quantum Northwest, qpod 2e). The mixture was cooled to the desired temperature (below the UCST) and left without stirring until the macroscopic phase separation was completed. Then, a syringe with a 22-gauge blunt tip needle was dipped into the solution to carefully withdraw an aliquot of the bottom 5CB-rich phase. The tip of the needle was subsequently lifted and immersed in the upper $\mathrm{MeOH}$-rich phase before a droplet was dispensed. Images of the drops were acquired with a digital microscope (Visio-Tek, IWT) that was attached to one port of the temperature controlled quartz cuvette. Image analysis was carried out using the Krüss Advance software as well as the pendent drop plug-in for ImageJ [25].

\section{Fabrication of microfluidics chips}

Microfluidic chips for the study of different flow regimes were fabricated as shown in Figure S2 in the Supplementary Information. Two different techniques were used for the sandwich architecture, namely UV-curable adhesive bonding of cyclo-olefin polymer sheets and thermal bonding of PVC. While the former resulted in higher optical quality chips, the latter enabled the realisation of more complex channel architectures, such as serpentines.

For thermally bonded chips, extruded sheets of PVC (Norva Plastics, $1.5 \mathrm{~mm}$ ) were cut in a CNC engraving machine (Roland,
EGX-400) according to the design. Both parts were then cleaned in water, dried in air, and subsequently aligned on top of each other to match the channel contours. The arrangement was fixed with two aluminium plates and then placed in an oven for 2 hours at $120^{\circ} \mathrm{C}$ for thermal bonding, i.e., above the glass transition temperature of PVC but below its melting point. Then, the PVC sandwich was left to cool to room temperature. A leak test was subsequently carried out by injecting water into the channels.

The fabrication of UV-bonded chips involved the use of optically transparent cyclo-olefin polymer sheets (Zeonor, 1060R, 1.6 $\mathrm{mm}$ in thickness) and an UV curing adhesive (Norland, Optical Adhesive 61). In this case, the cut and cleaned sheets were oxygen plasma treated for 1 minute to enhance the wetting with the adhesive. Subsequently, both parts were aligned with the activated surface facing each other and held in position with an adhesive tape. A droplet of the optical adhesive was placed at the edge of the device to prime the contact area between both parts. The sandwich was then cured by UV light (320-400 $\mathrm{nm}, 3$ Joules $/ \mathrm{cm}^{2}$ ) for 10 minutes and subsequently left in an oven at $50^{\circ} \mathrm{C}$ overnight to dry all residual adhesive and ensure maximum bonding strength.

\section{Flow regimes characterisation}

The formation of flow regimes was studied using the following experimental set-up (see Supplementary Information, Figure S3). Microfluidics chips were placed in a custom-built temperature-controlled chip holder that was connected with PTFE tubing. A binary 50/50 v/v liquid mixture of $\mathrm{MeOH}$ and $5 \mathrm{CB}$ $(2 \mathrm{ml})$ was prepared and mixed at $40^{\circ} \mathrm{C}$ in a temperature controlled quartz cuvette (Quantum Northwest, qpod 2e). Once the mixture was homogeneous and clear, the PTFE tubing was immersed in the solution. Flow rates were imposed with a 2.5 $\mathrm{ml}$ gas tight syringe connected to a syringe pump (Cronus, Sigma 1110) in withdrawal mode. The temperature of phase separation was controlled with a custom-built Peltier-based chip holder and the flow regimes were investigated with a digital microscope (Visio-Tek, IWT) that was placed on top of the microfluidic chips. Various rectangular channel architectures served in the course of this study, namely cross-sections of $500 \times 500 \mu^{2} ; 600 \times 600 \mu^{2} ; 700 \times 700 \mu \mathrm{m}^{2} ; 800 \times 800 \mu \mathrm{m}^{2}$ and $900 \times 900 \mu^{2}$. While thermally bonded PVC chips were used for the bulk of the study, high resolution images of the representative flow regimes of $\mathrm{MeOH}$ and $5 \mathrm{CB}$ were acquired using UV-cured Zeonor 1060R chips. In this case, a camera (Lumenera, Infinity 3-1) was connected to an optical microscope in transmission mode (Zeiss, Axio Scope A1) using differential interface contrast (PlasDIC) and recorded with the manufacturer's software (Lumenera, Infinity Analyze).

Experiments using binary liquid mixtures of water and 2,6lutidine (70/30 v/v) were carried out in an analogous way, $2 \mathrm{ml}$ of solution was prepared and mixed with a magnetic bar at room temperature. Once the mixture was homogeneous and 
clear, the PTFE tubing was immersed in the solution. The flow rate of the injected single-phase mixture was controlled with a $2.5 \mathrm{ml}$ gas tight syringe in a syringe pump (Cronus, Sigma 1110). The flow regimes were observed at separation temperatures of $38^{\circ} \mathrm{C}, 43^{\circ} \mathrm{C}, 48^{\circ} \mathrm{C}$ and $53^{\circ} \mathrm{C}$, respectively.

\section{Results and discussion}

To predict the behaviour of a binary liquid mixture in a microfluidic environment, it is necessary to understand the macroscopic $\mathrm{T}-\phi$ phase diagram of the blend. We have recently reported on the phase behaviour of $5 \mathrm{CB}$ and $\mathrm{MeOH}$ [26]. This mixture exhibits an UCST of $24.4^{\circ} \mathrm{C}$, a temperature range that can conveniently be accessed. Above the UCST, any composition is fully miscible and one single phase is observed. On the contrary, for a 50/50 v/v mixture below the UCST, twophases coexist, one rich in $\mathrm{MeOH}$ and the other rich in $5 \mathrm{CB}$. The phase composition can be determined via the Lever rule. The deeper the temperature quench below the UCST, the purer the phases become in their principal component.

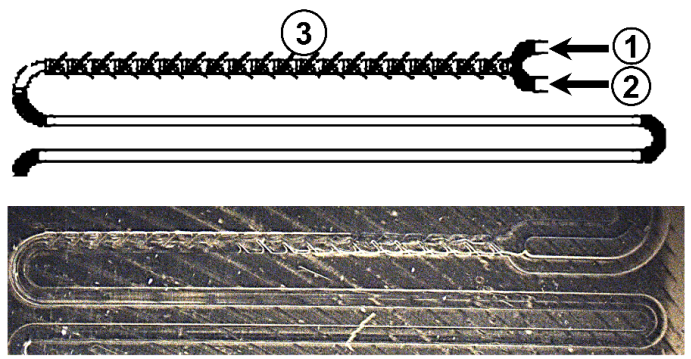

C

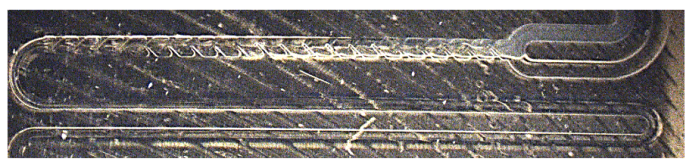

d

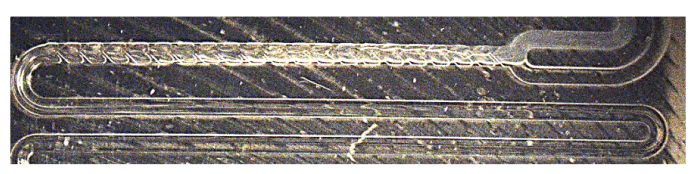

e

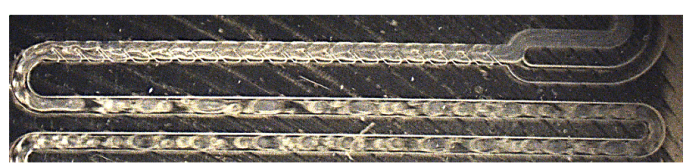

Figure 1 Temperature-dependent mixing of $5 \mathrm{CB}$ and $\mathrm{MeOH}$, infused at the top (a1) and bottom (a2) inlet, respectively. Passive mixing then occurs via a static micromixer with staggered oriented ridges (a3). (b-e) Snapshots of the microfluidic chip at various temperatures: $40^{\circ} \mathrm{C}$ (b); $35^{\circ} \mathrm{C}$ (c); $24{ }^{\circ} \mathrm{C}$ (d) and $12^{\circ} \mathrm{C}(\mathrm{e})$
The mixing of $5 \mathrm{CB}$ and $\mathrm{MeOH}$ was investigated in a static micromixer with staggered oriented ridges that was mounted to a Peltier-controlled chip holder (Figure 1a). At a chip temperature of $40^{\circ} \mathrm{C}$, both infused liquids, $5 \mathrm{CB}$ and $\mathrm{MeOH}$, exhibited a transparent isotropic state and a length of mixing of around $60 \mathrm{~mm}$ was required to observe a single homogeneous phase at a flow rate of $5 \mu \mathrm{l} / \mathrm{min}$ for each stream (Figure $1 \mathrm{~b}$ ). A similar behaviour was seen for a mixing temperature of $35^{\circ} \mathrm{C}$ (Figure 1c) and other temperatures above $24^{\circ} \mathrm{C}$. These microfluidic observations are in close agreement with the T- $\phi$ phase diagram established in bulk experiments [26]. The interaction length needed to develop a fully mixed phase is related to the diffusivity of $\mathrm{MeOH}$ and $5 \mathrm{CB}$ in laminar regimes, as has previously been studied for other miscible liquids [27]. As shown in Figure 1c-1e, the infused $5 C B$ changed its appearance from transparent to milky for chip temperatures below $35^{\circ} \mathrm{C}$, indicating the expected phase transition of the liquid crystal from isotropic to nematic to occur at temperatures below the UCST [28]. Once in contact with $\mathrm{MeOH}$, the nematic infusion turned transparent before becoming a single phase with the $\mathrm{MeOH}$ infusion. This behaviour is related to the fact that $5 \mathrm{CB}$ exhibits a significant decrease in the isotropic-to-nematic phase transition temperature upon enrichment with $\mathrm{MeOH}$, even if only small volume fractions of $\mathrm{MeOH}$ are added [26]. As described by the phase diagram, this effect is somewhat suppressed at lower temperatures when more $\mathrm{MeOH}$ is required to induce a nematic-to-isotropic transition. When mixing at $24^{\circ} \mathrm{C}$ (Figure $1 \mathrm{~d}$ ), a temperature slightly below the previously reported UCST of $24.4^{\circ} \mathrm{C}$, we observed a two-phase annular-type flow pattern. For temperatures of more than $3 \mathrm{~K}$ below the UCST, slug flow was observed after the fluid streams passed through the micromixer (Figure 1e). For a more detailed presentation of mixing behaviour in the range of $40^{\circ} \mathrm{C}$ to $12^{\circ} \mathrm{C}$, we refer to the Supplementary Information (Figure S4).

Mixers used in microfluidics are usually classified as passive mixers when no external energy is applied to create disturbance and enhance diffusion. In contrast, they are referred to as active mixers when an external energy is applied [29]. The setup used for this study introduces a combination of both, a static micromixer and active control of temperature. While the use of passive mixers has been studied in detail using microfluidics, the control of temperature to enhance mixing in microfluidics remains relatively unexplored [30]. Some of the few examples using active control of temperature for mixing include the use of two alternating heaters to enhance the natural convection of the fluids [19] and a thermal bubble actuated nozzle-diffuse micro pump to create a wave interface that increases the contact area between liquids to accelerate the diffusion process [31]. Both strategies enhance the diffusion of the liquid molecules by increasing the entropy of the system. In contrast, the strategy presented here relies on the highly temperaturedependent enthalpy of mixing of regular solutions. The approach therefore leverages on the intrinsic properties of the 
fluid mixture and offers unique opportunities for microfluidic applications. We note that mixing was also achieved in other chip architectures, including split-ring micromixers as well as serpentine arrangements, where no passive mixing element was included to reduce the length of the mixing unit.

A common limitation for mass transfer in liquid-liquid extraction with immiscible fluids is the need for the target compound to diffuse across the liquid-liquid interface [32]. The ability of this partially miscible binary liquid mixture to form a single phase above the UCST allows for a temporary elimination of the liquidliquid interface, thus simplifying the problem of mass transfer. The effect of cooling the heated single-phase mixture again below the UCST was studied in the following.

To study the influence of the temperature on the phase separation of the $5 \mathrm{CB} /$ methanol mixture, we injected the two fluids into a Zeonor microfluidic chip and investigated the phase separation with an optical microscope. When the temperature of the solution reached the critical temperature, spontaneous emulsification was observed, as indicated by the appearance of small $\mathrm{MeOH}$-rich droplets in a continuous $5 \mathrm{CB}$-rich phase. The emulsification was discernible in the microscope as an opaque phase due to the large amount of small droplets that scattered the light isotropically. This opaqueness was transient and disappeared once the droplets grew by coalescence. After the initial microscopic phase separation, both phases arranged in the microfluidic channels in a very regular manner.

To investigate the influence of the fluid velocity on the flow patterns, we systematically varied the cross-sections of the microfluidic channels $\left(500 \times 500 \mathrm{um}^{2}\right.$ to $\left.900 \times 900 \mu \mathrm{m}^{2}\right)$ as well as temperature $\left(5^{\circ} \mathrm{C}\right.$ to $\left.20^{\circ} \mathrm{C}\right)$ and flow rate $(1 \mu \mathrm{l} / \mathrm{min}$ to 100 $\mu \mathrm{l} / \mathrm{min}$ ). The two-phase system attained four different patterns, namely droplets, plugs, slugs, and annular flow, as shown in Figure 2. An overview of the obtained flow type as a function of superficial velocity $U$, estimated from the volume flow rate divided by the channel cross-sectional area, is shown for four different temperatures. At $20^{\circ} \mathrm{C}$, droplets were perceived at superficial velocities $\left(U_{\mathrm{MeOH}}, U_{5 \mathrm{CB}}\right)$ smaller than $0.0001 \mathrm{~m} / \mathrm{s}$. At higher superficial velocities, the formed droplets extended in diameter beyond the width of the channel, resulting in so-called plugs, that were surrounded by a continuous 5 CB phase. These plugs were observed for superficial velocities between 0.0001 $\mathrm{m} / \mathrm{s}$ and $0.0004 \mathrm{~m} / \mathrm{s}$. In close analogy to plug flow, we refer to slug flow, when the characteristic length of the methanol inclusion was at least 5 times the width of the channel [33]. Slug flow was found to occur for annular flow velocities between $0.0001 \mathrm{~m} / \mathrm{s}$ and $0.001 \mathrm{~m} / \mathrm{s}$. At superficial velocities above 0.001 $\mathrm{m} / \mathrm{s}$, annular flow was observed. For a temperature of $15^{\circ} \mathrm{C}$ and below, only plug flow was obtained for superficial velocities below $0.001 \mathrm{~m} / \mathrm{s}$. At higher superficial velocities we observed slug flow, but the transition was temperature-dependent, with slug extending to higher velocities at lower temperatures. We note that a similar trend in the formation of flow patterns has

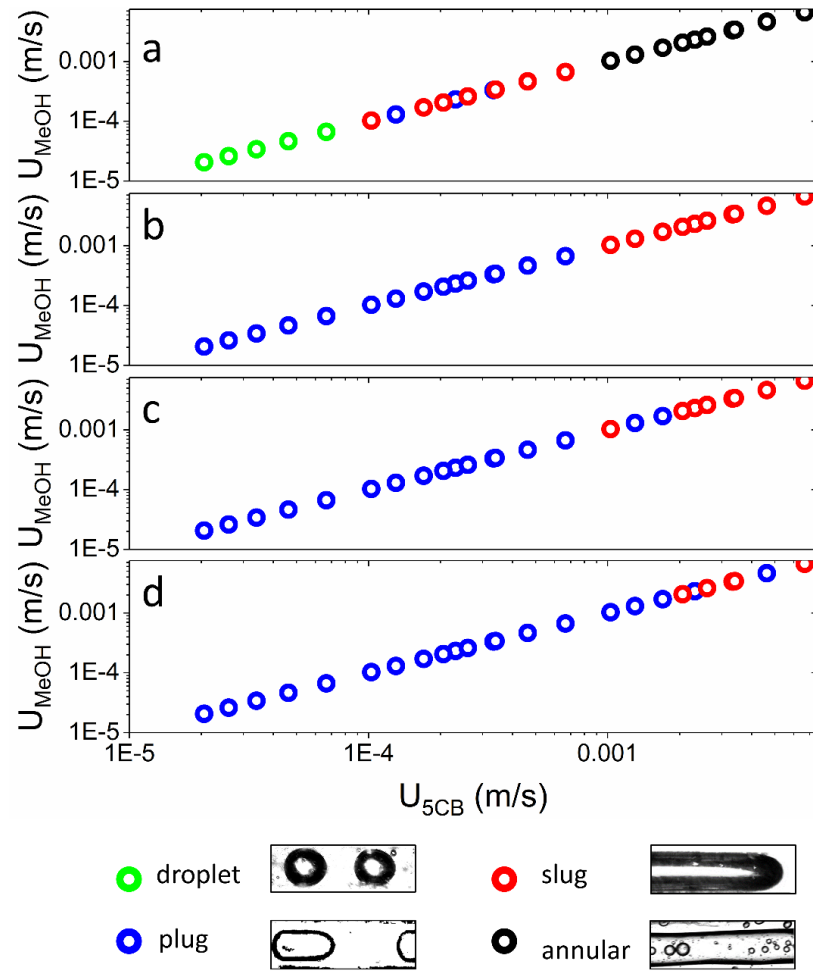

Figure 2 Superficial velocity of each phase after phase separation and corresponding two-phase regime. Four different regimes were observed, namely droplet, plug, slug and annular flow. a) $20^{\circ} \mathrm{C}$, b) $15^{\circ} \mathrm{C}$, c) $10^{\circ} \mathrm{C}$ and d) $5^{\circ} \mathrm{C}$.

been reported for an increasing superficial velocity of water and toluene [34] [35], which forms slug, slug-drop, a deformed interface, and parallel/annular flow, from low to high relative superficial velocities respectively. The estimated superficial velocity does not take into account the role of temperature and the consequent change in the liquids properties. In order to investigate the role of the temperature on the two-phase system, we measured the influence of temperature on the interfacial tension and viscosity of the binary liquid mixture. To generalize our findings, we then converted the data into dimensionless numbers, namely into Capillary (Ca) and Reynolds (Re) numbers. See Table 1 and Table 2 for a definition and the nomenclature, respectively.

Table 1 Dimensionless numbers used to compare flow regimes

\begin{tabular}{lcc} 
Dimensionless numbers & \\
\hline Capillary number & $\begin{array}{l}\text { viscous forces } \\
\text { interfacial forces }\end{array}$ & $\mathrm{Ca}=\frac{\mu \cdot \bar{v}}{\gamma}$ \\
\hline Reynolds number & $\frac{\text { inertial forces }}{\text { viscous forces }}$ & $\mathrm{Re}=\frac{\rho \cdot \bar{v} \cdot D}{\mu}$ \\
\hline
\end{tabular}

Table 2 Nomenclature

\begin{tabular}{|ccc|}
\hline$\mu$ & Dynamic viscosity & $\mathrm{kg} / \mathrm{m} \mathrm{s}$ \\
\hline $\bar{v}$ & Fluid velocity & $\mathrm{m} / \mathrm{s}$ \\
\hline$\gamma$ & Interfacial tension & $\mathrm{N} / \mathrm{m}$ \\
\hline$\rho$ & Density & $\mathrm{kg} / \mathrm{m}^{3}$ \\
\hline$d_{h}$ & Hydraulic diameter & $\mathrm{m}$ \\
\hline$\varepsilon_{5 C B}$ & Volumetric volume of $5 C B$ phase & \\
\hline
\end{tabular}




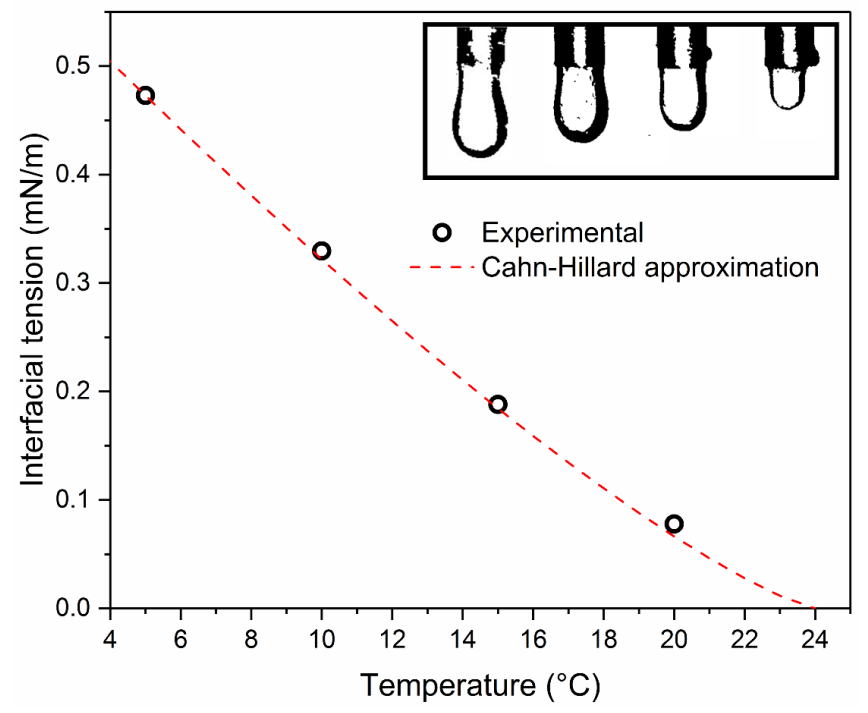

Figure 3 Interfacial tension as a function of temperature. Values were determined via pendant drop experiments of 5CB-rich droplets immersed in a $\mathrm{MeOH}$-rich phase. Representative droplets are shown in upper right box, from left to right at $5^{\circ} \mathrm{C}, 10^{\circ} \mathrm{C}, 15^{\circ} \mathrm{C}$ and $20^{\circ} \mathrm{C}$.

The interfacial tension was measured as a function of temperature using the pendant drop method, as shown in Figure 3. The measured interfacial tension of the 5CB-rich and $\mathrm{MeOH}$-rich phase was found to decrease as the temperature increased, from $0.47 \mathrm{mN} / \mathrm{m}$ at $5{ }^{\circ} \mathrm{C}$ to $0.077 \mathrm{mN} / \mathrm{m}$ at $20^{\circ} \mathrm{C}$. The pronounced temperature effect on the interfacial tension is also visually evident, when observing the 5 CB-rich droplet size in a $\mathrm{MeOH}$-rich medium, shown in the upper right box of Figure 3.

We relate this change in interfacial tension mainly to the compositional variation of the phases with temperature. At $20^{\circ} \mathrm{C}$, the mixture is very close to the UCST, and thus, both phases are more similar in composition, with around $30 \%$ of $\mathrm{MeOH} v / \mathrm{v}$ dissolved in the $5 \mathrm{CB}$-rich phase and a similar amount of $5 \mathrm{CB}$ mesogens dissolved in the $\mathrm{MeOH}$-rich phase. As the temperature decreases, the composition of each phase becomes richer in its principal component, leading to an expected $16 \%$ of $\mathrm{MeOH} v / \mathrm{v}$ dissolved in the $5 \mathrm{CB}$ rich phase at 5 ${ }^{\circ} \mathrm{C}$. The change in composition also changes the density of each phase, making the $5 \mathrm{CB}$-rich phase denser and $\mathrm{MeOH}$-rich phase lighter as the temperature decreases (shown in the Supplementary Information, Figure S5). We refer to earlier studies on the role of composition-dependent density contrast for the interfacial tension of partially miscible liquids [36] [37] [38]. The crucial effect of temperature on the interfacial tension is typical for regular solutions with an UCST and has been reported in a number of liquid-liquid systems, including cyclohexane/aniline [39], $\mathrm{MeOH} / \mathrm{n}$-hexane [40], and in polystyrene/cyclohexane [41]. We note that with composition being the dominant factor for the interfacial tension of partially miscible binary liquid mixtures, a solution with a lower critical solution temperature (LCST) should exhibit an inverse scaling with temperature. This is consistent with the reported behaviour of the binary system 2,6-lutidine and water (LCST $34.1^{\circ} \mathrm{C}$ ), for which the interfacial tension was found to increase from $0.007 \mathrm{mN} / \mathrm{m}$ at $34.2{ }^{\circ} \mathrm{C}$ to $0.8 \mathrm{mN} / \mathrm{m}$ at $60{ }^{\circ} \mathrm{C}$ [42].

The interfacial tension of a binary liquid mixture below the UCST is described by the Cahn-Hillard theory [37],

$$
\gamma=A_{0}\left(1-\frac{T}{T_{c}}\right)^{\mu}
$$

where $A_{0}$ is the critical amplitude of the interfacial tension near the critical point, $T$ is a temperature below the UCST, $T_{c}$ is the UCST and $\mu$ is the characteristic universal exponent with an empirical estimate of $\mu=1.26$ [43]. $A_{O}$ is specific to the liquidliquid mixture. An experimental value of $A_{O}=15.92 \pm 1.04 \mathrm{mN} / \mathrm{m}$ was obtained herein for $5 \mathrm{CB}$ and $\mathrm{MeOH}$ by applying the experimental values for the interfacial tension at different temperatures to the Cahn-Hillard equationm. When comparing the experimental results with the described approximation for the full temperature range in Figure 3, it is evident that CahnHillard model is appropriate to predict the interfacial tension below the UCST.

The dynamic viscosity of the $\mathrm{MeOH}$-rich and $5 \mathrm{CB}$-rich phase after phase separation was measured with a rheometer (Discovery, $\mathrm{HR}-3$ ) at $20^{\circ} \mathrm{C}, 15^{\circ} \mathrm{C}, 10^{\circ} \mathrm{C}$ and $5^{\circ} \mathrm{C}$. The binary liquid mixtures were heated to $40^{\circ} \mathrm{C}$ in a temperature controlled cuvette for uniform mixing and subsequently phase separated at a target temperature before aliquots of the upper and lower phase were separately extracted with a syringe. The obtained results are shown in the Supplementary Information (Figure S6). For the 5CB-rich phase, the measured dynamic viscosity was found to be inversely proportional to temperature, ranging from $26 \mathrm{mPa} * \mathrm{~s}$ at $5{ }^{\circ} \mathrm{C}$ to $7 \mathrm{mPa} * \mathrm{~s}$ at $20^{\circ} \mathrm{C}$. A less pronounced trend was observed for the $\mathrm{MeOH}$-rich phase, where the dynamic viscosity was determined to be $0.76 \mathrm{mPa}$ *s at $5{ }^{\circ} \mathrm{C}$ and $0.33 \mathrm{mPa}^{*} \mathrm{~s}$ at $20^{\circ} \mathrm{C}$, i.e. more than one order of magnitude lower than for the $5 \mathrm{CB}$-rich phase. We note that for 2,6lutidine/water, i.e. a fluid system with a LCST, the dynamic viscosity similarly decreased with increasing temperature (while the interfacial tension increased as noted above) [42].

An analogous interplay of viscosity and surface tension with temperature has been previously reported in microfluidic studies of immiscible liquid-liquid mixtures, such as water and mineral oil [20], water doped with $\mathrm{TiO}_{2}$ nanoparticles and mineral oil [21], water with Dynalene SF and perfluoroperhydrophenanthrene [22], as well as gelatin emulsion and mineral oil [23]. In all cases, the tunability of the interfacial tension and viscosity with temperature was found to be an important parameter for the control of droplet sizes and flow regimes. In comparison to previous studies, we investigate the behaviour of a partially miscible liquid-liquid mixture, which consequently offers a more pronounced variability of fluid 
parameters with temperature. While in previous studies the interfacial tension of water and oil was decreased from 52 $\mathrm{mN} / \mathrm{m}$ to $45 \mathrm{mN} / \mathrm{m}$ for a temperature increase from $25^{\circ} \mathrm{C}$ to 50 ${ }^{\circ} \mathrm{C}[21]$, we were able to tune the interfacial tension of the $5 \mathrm{CB}$ and $\mathrm{MeOH}$ mixture in a dynamic range of 0.47 to $0.077 \mathrm{mN} / \mathrm{m}$ for a temperature increase from $5{ }^{\circ} \mathrm{C}$ to $20^{\circ} \mathrm{C}$.

In order to understand the role of viscous, interfacial and inertial forces in the formation of the flow regimes, we plotted results for over 100 measurements at different temperatures, flow rates, and channel architectures using the parameters $\left(\mathrm{Re}_{\mathrm{MeOH}}{ }^{*} \mathrm{~d}_{\mathrm{h}} / \varepsilon_{\mathrm{MeOH}}\right)$ and $\left(\mathrm{Re}_{5 \mathrm{CB}} / \mathrm{Ca}_{5 \mathrm{CB}}\right)$ for $\mathrm{x}$ - and $\mathrm{y}$-axis, respectively [34]. Figure 4 shows the ratio between Reynolds and Capillary numbers of $5 \mathrm{CB}\left(\mathrm{Re}_{5 \mathrm{CB}} / \mathrm{Ca}_{5 \mathrm{CB}}\right)$ as a function of the parameter that relates Reynolds $\left(\operatorname{Re}_{\mathrm{MeOH}}\right)$, hydraulic number $\left(\mathrm{d}_{\mathrm{h}}\right)$ and volumetric flow fraction of $\mathrm{MeOH}\left(\varepsilon_{\mathrm{MeOH}}\right)$. A definition of both Reynolds and Capillary number with nomenclature of all variables is shown in Table 1 and 2, respectively. Further information on the construction of the flow regime map is shown in the Supplementary Information (Figure S7). As a general rule and for a given temperature, flow patterns grouped towards the left of the diagram are surface tension-dominated, while flow patterns grouped towards the right side of the diagram are inertia-dominated, with a transition zone in between. Indeed, formation of droplet flow was found favoured when viscous forces were dominant over interfacial tension and inertial forces, i.e., in the regime of lower surface tension. Consequently, the formation of plug and slug were favoured when the inertial or interfacial forces were increased, i.e., when the mixture was cooled or the flow rate was increased for a given channel cross section. These flow patterns were found grouped in a transition zone between surface tension and inertia dominated regions. On the other hand, annular flow was favoured when inertial forces were found dominant over interfacial tension and viscous forces, hence on the right side of the diagram.

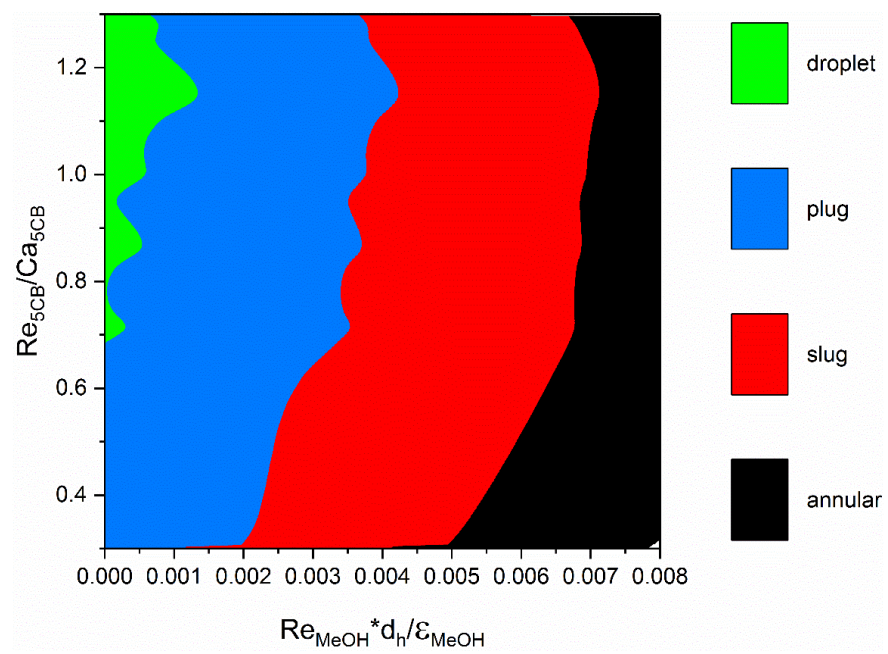

Figure 4 Map of observed flow regimes, plotted with parameters $\left(\operatorname{Re}_{\mathrm{MeOH}} * \mathrm{~d}_{\mathrm{h}} / \varepsilon_{\mathrm{MeOH}}\right)$ and $\left(R e_{5 \mathrm{CB}} / \mathrm{Ca}_{5 \mathrm{CB}}\right)$ for $\mathrm{x}$ - and y-axis, respectively.
We note that annular flow was only observed at $20^{\circ} \mathrm{C}$ and at relatively high flow rates, i.e. when the interfacial tension between the phases was minimal and the inertial forces of the $\mathrm{MeOH}$ phase were maximised.

Flow pattern studies have shown that superficial velocities of the phases and microchannel sizes play an important role in the observed flow regime. A direct consequence of using a temperature-responsive partially miscible binary liquid mixture in microfluidics is the introduction of temperature as a principal variable for the observed flow patterns. This is shown in Figure 5 , where active tuning of the system can be achieved by controlling the temperature at constant flow rates and channel cross section. At $5{ }^{\circ} \mathrm{C}$, where the interfacial tension of the mixture, density difference and dynamic viscosity of the phases are maximised, plug flow was observed (Figure 5a). As the temperature is increased to $10^{\circ} \mathrm{C}$, slug flow was observed, as shown in Figure $5 \mathrm{~b}$. At $15^{\circ} \mathrm{C}$, the flow transitioned into the annular regime (Figure $5 \mathrm{C}$ ) and at $20^{\circ} \mathrm{C}$, where the interfacial tension of the mixture, density difference and dynamic viscosity of the phases are minimized a fully developed annular flow was observed (Figure $5 \mathrm{~d}$ ). In consequence, partially miscible binary liquid mixtures offer a new route to actively control flow patterns in microfluidics by their responsiveness to temperature.

To demonstrate the broad applicability of this approach, we studied the temperature-dependent flow pattern for a partially miscible binary solution with a LCST, a $30 / 70 \mathrm{v} / \mathrm{v}$ mixture of 2,6lutidine/water. Three flow regimes were observed, namely plug, slug, and annular. Annular flow developed primarily at temperatures of $38{ }^{\circ} \mathrm{C}$ with fluid velocities between 0.003 and $0.006 \mathrm{~m} / \mathrm{s}$, while plug and slug flows were observed at temperatures varying above $38{ }^{\circ} \mathrm{C}$ and fluid velocities ranging from 0.00002 to $0.006 \mathrm{~m} / \mathrm{s}$. We refer to the Supplementary Information (Figure S8) for the corresponding flow map of the binary liquid mixture.

a

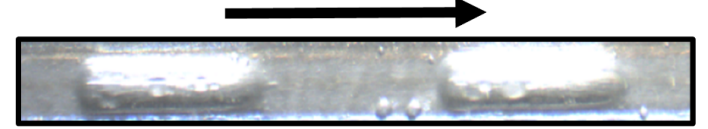

b

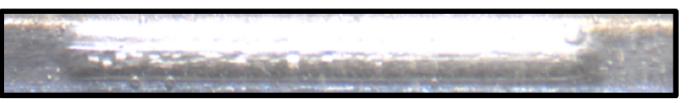

C
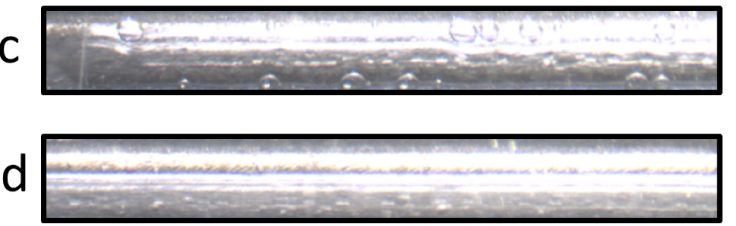

Figure 5 Temperature-induced tuning of different flow patterns. Observed flow patterns at a fixed flow rate $(70 \mu \mathrm{l} / \mathrm{min})$ and channel cross section $\left(700 \times 700 \mathrm{~mm}^{2}\right.$ ) with temperature as only variable: a) $5^{\circ} \mathrm{C}$, b) $\left.10^{\circ} \mathrm{C}, \mathrm{c}\right) 15^{\circ} \mathrm{C}$ and d) $20^{\circ} \mathrm{C}$. Black arrow indicates flow direction. 
The reported findings on the mixing and separation behaviour in microfluidics may be applicable to a wide range of other partially miscible liquid-liquid mixtures. Examples include binary blends of cyclohexane and aniline [39], methanol and n-hexane [40], polystyrene and cyclohexane [41], poly(methacrylamide) and water, poly(acrylamide-co-acrylonitrile) and water [44], 1n-butyl-3-methylimidazolium tetrafluoroborate and water [45], 1-hexyloxymethyl-3-methyl-imidazolium tetrafluoroborate and aliphatic or aromatic hydrocarbons [46], ionic liquids and alcohols [47], n-Propyl-tri-n-butylammonium iodide and water [48] as well as polymer ionic liquids with imidazolyl groups and water [49]. Possible applications of the highlighted systems are multi-fold and include drug delivery [50], catalysis [51], extraction and separation of bioactive compounds [52], micellemediated pre-concentration protocols [53] [54] and alternating current cloud point extraction [55].

\section{Conclusions}

In conclusion, we report on the microfluidic properties of partially miscible binary liquid mixtures with upper or lower critical solution temperature. In the case of an equal volume blend of the thermotropic liquid crystal 4-Cyano-4'pentylbiphenyl $(5 \mathrm{CB})$ and methanol $(\mathrm{MeOH})$, heating above the upper critical solution temperature of $24.4^{\circ} \mathrm{C}$ led to a homogeneous single phase, while subsequent cooling of the solution resulted in phase separation and development of welldefined two-phase flow regimes, namely droplet flow, plug flow, slug flow and annular flow. A flow pattern map was constructed to relate viscous, interfacial and inertial forces through dimensionless Reynolds and Capillary numbers. In surface tension dominated flow, the formation of droplets was found to be favoured, while higher inertial forces led to the formation of plug, slug and annular flow. Interestingly, the interfacial tension and the dynamic viscosity of the separated phases were found inversely proportional to temperature, allowing active tuning of the flow regime by temperature variation at constant channel architecture and flow rate. An analogous but inverted behaviour was observed for water and 2,6-lutidine, a binary liquid mixture with a lower critical solution temperature. The use of partially miscible solutions that exhibit a mixing and phase separation behaviour in an accessible temperature window is in our view a promising approach for temperature-controlled microfluidics, in particular in applications involving multi-stage processes and mass transfer across interfaces.

\section{Conflicts of interest}

There are no conflicts to declare.

\section{Acknowledgements}

This project received funding from the European Union's Horizon 2020 research and innovation programme under grant agreement No 633635 (DIACHEMO). MJF acknowledges funding by the EPSRC under a Doctoral Training Partnership. The authors are grateful for valuable support by Etienne Gianluca and Matej Janecek (EPFL) on pendant drop characterisation and analysis. MJF and SG acknowledge Dr Nicolay Dimov, Prof. Nicolas Szita and Prof. Asterios Gavriilidis (UCL) for help with microfluidic chip fabrication.

\section{References}

[1] A. Manz, D. Harrison, E. Verpoorte, J. Fettinger, A. Paulus, H. Lüdi and H. Widmer, Journal of Chromatography A, vol. 593, pp. 253-258, 1992.

[2] P. Dittrich and A. Manz, Nature Reviews Drug Discovery, vol. 5, pp. 210-218, 2006.

[3] C. Chin, V. Linder and S. Sia, Lab on a Chip, vol. 12, pp. 21182134, 2012.

[4] S. Sia and G. Whitesides, Electrophoresis, vol. 24, pp. 35633576, 2003.

[5] D. Breslauer, P. Lee and L. Lee, Molecular BioSystems, vol. 2, pp. 97-112, 2006.

[6] S. Takayama, E. Ostuni, P. LeDuc, K. Naruse, D. Ingber and G. Whitesides, Chemistry \& Biology, vol. 10, pp. 123-130, 2003.

[7] G. Whitesides, Nature, vol. 442, pp. 368-373, 2006.

[8] S. Xu, Z. Nie, M. Seo, P. Lewis, E. Kumacheva, H. Stone, P. Garstecki, D. Weibel, I. Gitlin and G. Whitesides, Angewandte Chemie, vol. 117, pp. 734-738, 2005.

[9] M. Kashid, A. Renken and L. Kiwi-Minsker, Chemical Engineering Science, vol. 66, pp. 3876-3897, 2011.

[10] K. Liu, R. Wu, Y. Chuang, H. Khoo, S. Huang and F. Tseng, Sensors, vol. 10, pp. 6623-6661, 2010.

[11] H. Song, J. Tice and R. Ismagilov, Angewandte Chemie, vol. 42, pp. 767-772, 2003.

[12] M. S. G. Nandagopal, R. Antony and N. Selvaraju, Microsystem Technologies, vol. 22, p. 349-356, 2014.

[13] N. Schwesinger, T. Frank and H. Wurmus, Journal of Micromechanics and Microengineering, vol. 6, pp. 99-102, 1996.

[14] A. Stroock, S. K. W. Dertinger, A. Ajdari, I. Mezic, H. A. Stone and G. M. Whitesides, Science, vol. 295, pp. 647-651, 2002. 
[15] H. Song, M. R. Bringer, J. D. Tice, C. J. Gerdts and R. F. Ismagilov, Applied Physics Letters, vol. 83, pp. 4664-4666, 2003.

[16] D. Ahmed, X. Mao, J. Shi, B. K. Juluri and T. J. Huang, Lab on a Chip, vol. 9, p. 2738-2741, 2009.

[17] L.-H. Lu, K. S. Ryu and C. Liu, Springer Netherlands, pp. 28-30, 2001.

[18] C. Yung, J. Fiering, A. Mueller and D. Ingber, Lab on a Chip, vol. 9, pp. 1171-1177, 2009.

[19] S.-K. Kim, F. Wang, M. A. Burns and K. Kurabayashi, Analytical Chemistry, vol. 81, pp. 4510-4516, 2009.

[20] N.-T. Nguyen, T.-H. Ting, Y.-F. Yap, T.-N. Wong and J. C.-K. Chai, Applied Physics Letters, vol. 91, 2007.

[21] S. M. Sohel Murshed, S. H. Tan and N. T. Nguyen, Journal of Physics D: Applied Physics, vol. 41, 2008.

[22] C. A. Stan, S. K. Tang and G. M. Whitesides, Analytical Chemistry, vol. 81, p. 2399-2402, 2009.

[23] C. Yeh, K. Chen and Y. Lin, Microfluidics and Nanofluidics, vol. 15, pp. 775-784, 2013

[24] A. W. Francis, Critical Solution Temperatures, vol. 31, Washington, D. C.: American Chemical Society, 1961.

[25] A. Daerr and A. Mogne, Journal of Open Research Software, vol. 4, p. e3, 2016.

[26] L. A. Serrano Gonzalez, M. J. Jara Fornerod, Y. Yang, S. Gaisford, F. Stellacci and S. Guldin, Soft Matter, vol. 14, pp. 4615-4620, 2018

[27] J. Atencia and D. J. Beebe, Nature, vol. 437, pp. 648-655, 2005.

[28] G. W. Gray, K. J. Harrison and J. A. Nash, Electronics Letters, vol. 9, pp. 130-131, 1973.

[29] N.-T. Nguyen and Z. Wu, Journal of Micromechanics and Microengineering, vol. 15, pp. R1-R16, 2005.

[30] K. Ward and Z. H. Fan, Journal of Micromechanics and Microengineering, vol. 25, 2015.

[31] J.-H. Tsai and L. Lin, Sensors and Actuators A: Physical, Vols. 97-98, pp. 665-671, 2002.

[32] A. L. Dessimoz, A. Renken and L. Kiwi-Minsker, Chemical Engineering Science, p. 4035-4044, 2008.

[33] H. Foroughi and M. Kawaji, International Journal of Multiphase Flow, vol. 37, p. 1147-1155, 2011.
[34] M. Kashid and L. Kiwi-Minsker, Chemical Engineering and Processing: Process Intensification, vol. 50, pp. 972-978, 2011.

[35] N. Kashid, A. Renken and L. Kiwi-Minsker, Industrial \& Engineering Chemistry Research, vol. 50, p. 6906-6914, 2011.

[36] J. S. Rowlinson, Journal of Statistical Physics, vol. 20, p. 197200, 1979.

[37] J. W. Cahn and J. E. Hilliard, The Journal of Chemical Physics, vol. 28, p. 258-267, 1958.

[38] M. E. Boudh-Hir and G. A. Mansoori, Physica A: Statistical Mechanics and Its Applications, vol. 179, pp. 219-231, 1991.

[39] D. Atack and O. K. Rice, Discussions of the Faraday Society, vol. 15, pp. 210-218, 1953.

[40] S. Abbas, J. Satherley and R. Penfold, Journal of the Chemical Society, Faraday Transactions, vol. 93, p. 2083-2089, 1997.

[41] J. S. Choi and Y. C. Bae, Journal of Chemical \& Engineering Data, vol. 61, p. 4157-4163, 2016.

[42] C. A. Grattoni, , R. A. Dawe, C. Y. Seah and J. D. Gray, Journal of Chemical \& Engineering Data, vol. 38, p. 516-519, 1993.

[43] J. S. Rowlinson and B. Widom, Molecular Theory of Capillarity, Mineola, New York: Dover Publications, 1982.

[44] J. Seuring and S. Agarwal, Macromolecules, vol. 45, p. 39103918, 2012.

[45] J. E. L. Dullius, P. A. Z. Suarez, S. Einloft, R. F. de Souza, J. Dupont, J. Fischer and A. De Cian, Organometallics, vol. 17, p. 815-819, 1998.

[46] U. Domańska and A. Marciniak, The Journal of Chemical Thermodynamics, vol. 37, p. 577-585, 2005.

[47] J. M. Crosthwaite, M. J. Muldoon, S. N. V. K. Aki, E. J. Maginn and J. F. Brennecke, The Journal of Physical Chemistry B, vol. 110, p. 9354-9361, 2006.

[48] M. Kleemeier, W. Schröer and H. Weingärtner, Journal of Molecular Liquids, vol. 73-74, p. 501-511, 1997.

[49] H. Yoshimitsu, A. Kanazawa, S. Kanaoka and S. Aoshima, Macromolecules, vol. 45, p. 9427-9434, 2012.

[50] D. Schmaljohann, Advanced Drug Delivery Reviews, vol. 58, p. 1655-1670, 2006.

[51] Y. Qiao, W. Ma, N. Theyssen, C. Chen and Z. Hou, Chemical Reviews, vol. 117, p. 6881-6928, 2017. 
[52] S. P. M. Ventura, F. A. e Silva, M. V. Quental, M. G. Freire and J. A. P. Couthino, Chemical Reviews, vol. 117, p. 6984-7052, 2017.

[53] C. D. Stalikas, TrAC Trends in Analytical Chemistry, vol. 21, pp. 343-345, 2002.

[54] C. Bordier, The Journal of Biological Chemistry, vol. 256, pp. 1604-1607, 1981.

[55] N. Sasaki, K. Hosokawa and M. Maeda, Lab on a Chip, vol. 9, pp. 1160-1170, 2009. 